\title{
BEREZIN-TOEPLITZ QUANTIZATION
}

AND BEREZIN TRANSFORM

MARTIN SChLICHENMAIER

September 2000, Manuskripte Nr. 25:7 


\title{
BEREZIN-TOEPLITZ QUANTIZATION AND BEREZIN TRANSFORM
}

\author{
MARTIN SCHLICHENMAIER
}

\begin{abstract}
In this lecture results on the Berezin-Toeplitz quantization of arbitrary compact quantizable Kähler manifolds are presented. These results are obtained in joint work with M. Bordemann and E. Meinrenken. The existence of the Berezin-Toeplitz deformation quantization is also covered. Recent results obtained in joint work with A. Karabegov on the asymptotic expansion of the Berezin transform for arbitrary quantizable compact Kähler manifolds are explained. As an application the asymptotic expansion of the Fubini-Study fundamental form under the coherent state embedding is considered. Some comments on the dynamics of the quantum operators are given.

(This is an extended write-up of an invited lecture presented at the workshop "Asymptotic properties of time evolutions in classical and quantum systems", September 13-17, 1999, Bologna, Italy.)
\end{abstract}

\section{INTRODUCTION}

In this lecture I would like to present results on the quantization of compact Kähler manifolds. More precisely, I consider the Berezin-Toeplitz quantization and its related BerezinToeplitz deformation quantization. These quantization schemes are very much adapted to the situation where the phase-space manifold is a Kähler manifold $(M, \omega)$. Whereas the basic objects can be defined for arbitrary Kähler manifolds the methods employed in the proofs of the theorems rely on the compactness of $M$. This is the reason that I will restrict the presentation to compact Kähler manifolds from the beginning.

The basic set-up is the set-up of geometric quantization. Firstly, this means that the Kähler form $\omega$ endows the algebra of differentiable functions on $M$ with a Poisson structure. Secondly, we assume that the Kähler manifold is a quantizable Kähler manifold, i.e. it admits a holomorphic quantum line bundle with curvature essentially equal to the Kähler form. To every differentiable function on $M$ there exists a family of Toeplitz operators $T_{f}^{(m)}\left(m \in \mathbb{N}_{0}\right)$ operating on the space of global holomorphic sections of the $m$-th tensor power of the quantum line bundle. In this way one obtains the Berezin-Toeplitz quantization. The set-up is explained in detail in Section 2. It was shown by Bordemann, Meinrenken and myself [4] that the Berezin-Toeplitz quantization has the correct semi-classical behaviour for $m \rightarrow \infty$. The results are presented in Section 3.

The proofs essentially use the theory of generalized Toeplitz operators developed by Boutet de Monvel and Guillemin [5]. A sketch of the techniques involved is given in Section 4. With

Date: September 23, 2000.

Key words and phrases. deformation quantization, Kähler manifolds, Berezin transform, semi-classical approximation, Berezin-Toeplitz quantization, geometric quantization, Toeplitz operators, Bergman kernel. 
the same techniques it is possible to construct a formal deformation quantization (also called a star product) [18],[20],[22]. The results are also presented in Section 3.

As it is shown in joint work with A. Karabegov [14] the obtained star product has some nice properties (i.e. "separation of variables", see below). On the way to prove these properties it is shown that the Berezin transform has an asymptotic expansion of certain type. This results is of independent interest. The Berezin transform associates to the function $f$ via its Toeplitz operator $T_{f}^{(m)}$ and the covariant Berezin symbol $\sigma\left(T_{f}^{(m)}\right)$ of this operator another differentiable function $I^{(m)}(f)$. Its asymptotics for $m \rightarrow \infty$ carries important information. Some of the results obtained in [14] are explained in Section 5 and in the appendix.

Applications of the asymptotic expansions are given in Section 6. Beside another proof of one part of the correct semi-classical behaviour of the Berezin-Toeplitz quantization scheme, the asymptotic expansion of the pull-back of the Fubini-Study form of the projective space in which the manifold is embedded using the global holomorphic sections of the $m$-th tensor power of the quantum line bundle for $m \rightarrow \infty$ is identified. The existence of such an asymptotic expansion was proven by Zelditch [26]. Here, we show that it can be identified (after assigning to the asymptotic expansion a formal form) with the classifying Karabegov form for the "opposite dual" star product to the Berezin-Toeplitz star product.

The results presented are mainly dealing with kinematics. It is possible to study dynamics by considering operators on the collection of quantum Hilbert spaces given by the space of global holomorphic sections of the quantum line bundle and its tensor powers. Some comments can be found in Section 7. Further work is in progress. There are some results in this field due to Zelditch [25], and others.

Finally, I like to thank the organizers of the workshop S. Graffi and A. Martinez for the kind invitation to present these results, and them and other participants for stimulating discussions. I also like to thank M. Engliš for bringing the work of Zelditch to my attention.

\section{THE SET-UP}

In the following we consider only phase-space manifolds which carry the structure of a compact Kähler manifold $(M, \omega)$. In particular, we take as symplectic form the Kähler form $\omega$ which is a non-degenerate closed positive $(1,1)$-form. If the complex dimension of $M$ is $n$ then the Kähler form $\omega$ can be written with respect to local holomorphic coordinates $\left\{z_{i}\right\}_{i=1, \ldots, n}$ as

$$
\omega=\mathrm{i} \sum_{i, j=1}^{n} g_{i j}(z) d z_{i} \wedge d \bar{z}_{j},
$$

with local functions $g_{i j}(z)$ such that the matrix $\left(g_{i j}(z)\right)_{i, j=1, \ldots, n}$ is hermitian and positive definite.

Denote by $C^{\infty}(M)$ the algebra of complex-valued (arbitrary often) differentiable functions with associative product the point-wise multiplication. Using the Kähler form $\omega$ one assigns to every $f \in C^{\infty}(M)$ its Hamiltonian vector field $X_{f}$ and to every pair of functions $f$ and $g$ the Poisson bracket $\{.,$.$\} via$

$$
\omega\left(X_{f}, \cdot\right)=d f(\cdot), \quad\{f, g\}:=\omega\left(X_{f}, X_{g}\right) .
$$


With the Poisson bracket $C^{\infty}(M)$ becomes a Poisson algebra. This means that $\{.,$.$\} defines$ an additional Lie algebra structure on $C^{\infty}(M)$ which fulfills as compatibility condition with the associative structure the Leibniz rule $\{f g, h\}=f\{g, h\}+\{f, h\} g$.

The Kähler manifold $(M, \omega)$ is called quantizable if there exists an associated quantum line bundle $(L, h, \nabla)$, where $L$ is a holomorphic line bundle $L$ over $M, h$ a Hermitian metric on $L$, and $\nabla$ a connection compatible with the metric $h$ and the complex structure (and hence uniquely fixed), such that the curvature form of the connection and the Kähler form $\omega$ of the manifold are related as

$$
\operatorname{curv}_{L, \nabla}(X, Y):=\nabla_{X} \nabla_{Y}-\nabla_{Y} \nabla_{X}-\nabla_{[X, Y]}=-\mathrm{i} \omega(X, Y) .
$$

Equation (2.3) is called the quantization condition. If the metric is represented as a function $\hat{h}$ with respect to local complex coordinates and a local holomorphic frame of the bundle the quantization condition reads as i $\bar{\partial} \partial \log \hat{h}=\omega$.

Example. The Riemann sphere, (i.e. the complex projective line) $\mathbb{P}(\mathbb{C})=\mathbb{C} \cup\{\infty\} \cong S^{2}$. With respect to the quasi-global coordinate $z$ the form can be given as

$$
\omega=\frac{\mathrm{i}}{(1+z \bar{z})^{2}} d z \wedge d \bar{z} \text {. }
$$

The quantum line bundle $L$ is the hyperplane bundle. For the Poisson bracket one obtains

$$
\{f, g\}=\mathrm{i}(1+z \bar{z})^{2}\left(\frac{\partial f}{\partial \bar{z}} \cdot \frac{\partial g}{\partial z}-\frac{\partial f}{\partial z} \frac{\partial g}{\partial \bar{z}}\right) .
$$

Example. The (complex-) onedimensional torus $M$. Up to isomorphy it can be given as $M=\mathbb{C} / \Gamma_{\tau}$ where $\Gamma_{\tau}:=\{n+m \tau \mid n, m \in \mathbb{Z}\}$ is a lattice with $\operatorname{Im} \tau>0$. As Kähler form we take

$$
\omega=\frac{\mathrm{i} \pi}{\operatorname{Im} \tau} d z \wedge d \bar{z},
$$

with respect to the coordinate $z$ on the covering space $\mathbb{C}$. Clearly this form is invariant under $\Gamma_{\tau}$ and hence well-defined on $M$. The corresponding quantum line bundle is the theta line bundle of degree 1, i.e. the bundle whose global sections are scalar multiples of the Riemann theta function. For the Poisson bracket one obtains

$$
\{f, g\}=\mathrm{i} \frac{\operatorname{Im} \tau}{\pi}\left(\frac{\partial f}{\partial \bar{z}} \cdot \frac{\partial g}{\partial z}-\frac{\partial f}{\partial z} \frac{\partial g}{\partial \bar{z}}\right) .
$$

The quantization condition (2.3) implies that $L$ is a positive line bundle. By the Kodaira embedding theorem $L$ is ample, which says that a certain tensor power $L^{m_{0}}$ of $L$ is very ample, i.e. the global holomorphic sections of $L^{m_{0}}$ can be used to embed the phase space manifold $M$ into projective space (the embedding will be explained in a second). In the following we will assume $L$ already to be very ample. If $L$ is not very ample we choose $m_{0} \in \mathbb{N}$ such that the bundle $L^{m_{0}}$ is very ample and take this bundle as quantum line bundle with respect to the Kähler form $m_{0} \omega$ on $M$. The underlying complex manifold structure is not changed.

We choose local holomorphic coordinates $z$ for $M$ and a local holomorphic frame $e(z)$ for the bundle $L$. After these choices the basis $s_{0}, s_{1}, \ldots, s_{N}$ for $\Gamma_{h o l}(M, L)$, the space of 
global holomorphic sections of the bundle $L$, can be uniquely described by local holomorphic functions $\hat{s}_{0}, \hat{s}_{1}, \ldots, \hat{s}_{N}$ defined via $s_{j}(z)=\hat{s}_{j}(z) e(z)$. The embedding is given by the map

$$
M \hookrightarrow \mathbb{P}^{N}(\mathbb{C}), \quad z \mapsto \phi(z)=\left(\hat{s}_{0}(z): \hat{s}_{1}(z): \cdots: \hat{s}_{N}(z)\right)
$$

Note that the point $\phi(z)$ in projective space neither depends on the choice of local coordinates nor on the choice of the local frame for the bundle $L$. A different choice of basis is equivalent unter a $\operatorname{PGL}(N, \mathbb{C})$ action on the embedding space. By this embedding we see that quantizable compact Kähler manifolds are as complex manifolds projective algebraic manifolds. The converse is also true, see [20],[1]. Note that the embedding is an embedding as complex manifolds not an isometric embedding as Kähler manifolds.

To introduce a scalar product on the space of sections we take the Liouville form $\Omega=\frac{1}{n !} \omega^{\wedge n}$ as volume form on $M$ and set for the scalar product and the norm

$$
\langle\varphi, \psi\rangle:=\int_{M} h(\varphi, \psi) \Omega, \quad\|\varphi\|:=\sqrt{\langle\varphi, \varphi\rangle},
$$

on the space $\Gamma_{\infty}(M, L)$ of global $C^{\infty}$-sections. Let $\mathrm{L}^{2}(M, L)$ be the $\mathrm{L}^{2}$-completion of $\Gamma_{\infty}(M, L)$, and $\Gamma_{\text {hol }}(M, L)$ be its (due to the compactness of $M$ ) finite-dimensional closed subspace of global holomorphic sections. Let $\Pi: \mathrm{L}^{2}(M, L) \rightarrow \Gamma_{h o l}(M, L)$ be the projection.

Definition 2.1. For $f \in C^{\infty}(M)$ the Toeplitz operator $T_{f}$ is defined to be

$$
T_{f}:=\Pi(f): \quad \Gamma_{h o l}(M, L) \rightarrow \Gamma_{h o l}(M, L) .
$$

In words: One takes a holomorphic section $s$ and multiplies it with the differentiable function $f$. The resulting section $f \cdot s$ will only be differentiable. To obtain a holomorphic section one has to project it back on the subspace of holomorphic sections.

The linear map

$$
T: C^{\infty}(M) \rightarrow \operatorname{End}\left(\Gamma_{h o l}(M, L)\right), \quad f \rightarrow T_{f},
$$

is the Berezin-Toeplitz quantization map. Because in general $T_{f} T_{g}=\Pi(f \cdot) \Pi(g \cdot) \Pi \neq$ $\Pi(f g \cdot) \Pi=T_{f g}$, it is neither a Lie algebra homomorphism nor an associative algebra homomorphism. The Berezin-Toeplitz quantization is a map from the commutative algebra of functions to a noncommutative finite-dimensional (matrix) algebra. The finite-dimensionality is due to compactness of $M$. A lot of classical information will get lost. To recover this information one should consider not just the bundle $(L, \nabla, h)$ alone but all its tensor powers $\left(L^{m}, \nabla^{(m)}, h^{(m)}\right)$ (we use the notation $L^{m}:=L^{\otimes m}$ ) and apply the above constructions for every $m \in \mathbb{N}_{0}$. Note that if $\hat{h}$ corresponds to the metric $h$ with respect to a local holomorphic frame $e$ of the bundle $L$ then $\hat{h}^{m}$ corresponds to the metric $h^{(m)}$ with respect to the frame $e^{\otimes m}$ for the bundle $L^{m}$. In this way one obtains a family of finite-dimensional(matrix) algebras and a family of maps

$$
T^{(m)}: C^{\infty}(M) \rightarrow \operatorname{End}\left(\Gamma_{h o l}\left(M, L^{m}\right)\right), \quad f \rightarrow T_{f}^{(m)}=\Pi^{(m)}(f \cdot), m \in \mathbb{N}_{0} .
$$

This infinite family should in some sense "approximate" the algebra $C^{\infty}(M)$. 


\section{The SEMi-Classical Behaviour and the Berezin-Toeplitz Deformation QUANTIZATION}

For the rest of the lecture let $(M, \omega)$ be a fixed quantizable compact Kähler manifold and $(L, h)$ a fixed very ample quantum line for it. Because the connection is uniquely fixed I will drop it in the notation. Denote for $f \in C^{\infty}(M)$ by $\|f\|_{\infty}$ the sup-norm of $f$ on $M$ and by

$$
\left\|T_{f}^{(m)}\right\|:=\sup _{\substack{s \in \Gamma_{h o l}\left(M, L^{m}\right) \\ s \neq 0}} \frac{\left\|T_{f}^{(m)} s\right\|}{\|s\|}
$$

the operator norm with respect to the analogue of $(2.9)$ on $\Gamma_{h o l}\left(M, L^{m}\right)$. The following theorem was shown in 1994.

Theorem 3.1. [Bordemann, Meinrenken, Schlichenmaier]

(a) For every $f \in C^{\infty}(M)$ there exists $C>0$ such that

$$
\|f\|_{\infty}-\frac{C}{m} \leq\left\|T_{f}^{(m)}\right\| \leq\|f\|_{\infty} .
$$

In particular, $\lim _{m \rightarrow \infty}\left\|T_{f}^{(m)}\right\|=\|f\|_{\infty}$.

(b) For every $f, g \in C^{\infty}(M)$

$$
\left\|m \mathrm{i}\left[T_{f}^{(m)}, T_{g}^{(m)}\right]-T_{\{f, g\}}^{(m)}\right\|=O\left(\frac{1}{m}\right) .
$$

(c) For every $f, g \in C^{\infty}(M)$

$$
\left\|T_{f}^{(m)} T_{g}^{(m)}-T_{f \cdot g}^{(m)}\right\|=O\left(\frac{1}{m}\right) .
$$

These results are contained in Theorem 4.1, 4.2, resp. in Section 5 in [4]. Note that (c) generalizes trivially to finitely many functions.

In [3] the notion of $L_{\alpha}$, resp. $g l(N)$, resp. $s u(N)$ quasi-limit was used introduced. It is related to the concept of continuous fields of $C^{*}$-algebras and the notion of strict quantization (see [15],[17]) for the definitions). It was conjectured in [3] that for every compact Kähler manifold the Poisson algebra of functions is a $g l(N)$ quasi-limit. This was proved in [4]. The result is of special interest in the theory of membranes. It follows also that the Berezin-Toeplitz quantization is a strict quantization.

Remark. There is another geometric concept of quantization, the geometric quantization introduced by Kostant and Souriau. Due to a result of Tuynman [23] (see also [3] for a coordinate independent proof) for compact Kähler manifolds both quantization schemes have the same semi-classical behaviour. More precisely, if $Q_{f}^{(m)}$ denotes the well-known operator of geometric quantization with respect to Kähler polarization, and $\Delta$ is the Laplacian with respect to the Kähler metric given by $\omega$ then $Q_{f}^{(m)}=\mathrm{i} \cdot T_{f-\frac{1}{2 m} \Delta f}^{(m)}$.

With the help of Toeplitz operators it is possible to construct a deformation quantization. A deformation quantization is given by a star product. I will use both terms interchangeable. To fix the notation and the factors of $\mathrm{i}$ let me recall the definition of a star product. Let $\mathcal{A}=C^{\infty}(M)[[\nu]]$ be the algebra of formal power series in the variable $\nu$ over the algebra 
$C^{\infty}(M)$. A product $\star$ on $\mathcal{A}$ is called a (formal) star product for $M$ (or for $C^{\infty}(M)$ ) if it is an associative $\mathbb{C}[[\nu]]$-linear product which is $\nu$-adically continuous such that

1. $\quad \mathcal{A} / \nu \mathcal{A} \cong C^{\infty}(M)$, i.e. $f \star g \bmod \nu=f \cdot g$,

2. $\frac{1}{\nu}(f \star g-g \star f) \bmod \nu=-\mathrm{i}\{f, g\}$,

where $f, g \in C^{\infty}(M)$.

We can write

$$
f \star g=\sum_{j=0}^{\infty} C_{j}(f, g) \nu^{j},
$$

with $C_{j}(f, g) \in C^{\infty}(M)$. The $C_{j}$ should be $\mathbb{C}$-bilinear in $f$ and $g$. The conditions 1 . and 2 . can be reformulated as

$$
C_{0}(f, g)=f \cdot g, \quad \text { and } \quad C_{1}(f, g)-C_{1}(g, f)=-\mathrm{i}\{f, g\} .
$$

By the $\nu$-adic continuity (3.5) fixes $\star$ on $\mathcal{A}$.

Theorem 3.2. There exists a unique (formal) star product $\star_{B T}$ for $M$

$$
f \star_{B T} g:=\sum_{j=0}^{\infty} \nu^{j} C_{j}(f, g), \quad C_{j}(f, g) \in C^{\infty}(M),
$$

in such a way that for $f, g \in C^{\infty}(M)$ and for every $N \in \mathbb{N}$ we have with suitable constants $K_{N}(f, g)$ for all $m$

$$
\left\|T_{f}^{(m)} T_{g}^{(m)}-\sum_{0 \leq j<N}\left(\frac{1}{m}\right)^{j} T_{C_{j}(f, g)}^{(m)}\right\| \leq K_{N}(f, g)\left(\frac{1}{m}\right)^{N} .
$$

This theorem has been proven immediately after [4] was finished. It has been announced in $[20],[19]$ and the proof was written up in German in [18]. A complete proof published in English can be found in [22].

If we write

$$
T_{f}^{(m)} \cdot T_{g}^{(m)} \sim \sum_{j=0}^{\infty}\left(\frac{1}{m}\right)^{j} T_{C_{j}(f, g)}^{(m)} \quad(m \rightarrow \infty)
$$

in the following we will always assume the strong and precise statement of (3.8). The same is assumed for other asymptotic formulas appearing further down in the article.

In the proof of the above theorems the concept of Toeplitz structure due to Boutet de Monvel and Guillemin [5] is employed. In Section 4 I will explain this structure and give a sketch of the proof of Relation (3.3). In Section 6.1 I will give a proof of Relation (3.2) using results on the Berezin transform. In the original article [4] a different proof was presented.

A further investigation shows that the Berezin-Toeplitz deformation quantization has some important properties (see [22] for definitions and proofs). It is "null on constants", i.e. $1 \star$ $f=f \star 1=f$. It is selfadjoint, i.e. $\overline{f \star f}=\bar{g} \star \bar{f}$. It admits a trace. As it is shown in [14] it is local and fulfills the "separation of variables" property. Locality means that $\operatorname{supp} C_{j}(f, g) \subseteq \operatorname{supp} f \cap \operatorname{supp} g$ for all $f, g \in C^{\infty}(M)$. From the locality property it follows that the $C_{j}$ are bidifferential operators and that the global star product defines for every open subset $U$ of $M$ a star product for the Poisson algebra $C^{\infty}(U)$. "Separation of variables" 
[13] means that $f \star k=f \cdot k$ and $k \star g=k \cdot g$ for (locally defined) holomorphic functions $g$, antiholomorphic functions $f$, and arbitrary functions $k$. Note that in Karabegov's notation the rôles of the holomorphic and antiholomorphic functions is switched.

\section{The Toeplitz Structure}

In [4] the set-up for the proof of the approximation results was given. Let me recall for further reference the main definitions. A more detailed exposition can be found in [18]. Take $(U, k):=\left(L^{*}, h^{-1}\right)$ the dual of the very ample quantum line bundle with dual metric $k$, $Q:=\{\lambda \in U \mid k(\lambda, \lambda)=1\}$ the unit circle bundle inside $U$, and $\tau: Q \rightarrow M$ the projection. Note that for the projective space with quantum line bundle the hyperplane section bundle $H$, the bundle $U$ is just the tautological bundle. Its fibre over the point $z \in \mathbb{P}^{N}(\mathbb{C})$ consists of the line in $\mathbb{C}^{N+1}$ which is represented by $z$. In particular, for the projective space the total space of $U$ with the zero section removed can be identified with $\mathbb{C}^{N+1} \backslash\{0\}$. The same picture remains true for the via the very ample quantum line bundle in projective space embedded manifold $M$. The quantum line bundle will be the pull-back of $H$ (i.e. its restriction to the embedded manifold) and its dual is the pull-back of the tautological bundle.

In the following we use $E \backslash 0$ to denote the total space of a vector bundle $E$ with the image of the zero section removed. Starting from the function $\hat{k}(\lambda):=k(\lambda, \lambda)$ on $U$ we define $\tilde{a}:=\frac{1}{2 \mathrm{i}}(\partial-\bar{\partial}) \log \hat{k}$ on $U \backslash 0$ (with respect to the complex structure on $U$ ) and denote by $\alpha$ its restriction to $Q$. Now $d \alpha=\tau^{*} \omega$ (with $d=d_{Q}$ ) and $\mu=\frac{1}{2 \pi} \tau^{*} \Omega \wedge \alpha$ is a volume form on $Q$. We have for $f \in C^{\infty}(M)$ the relation $\int_{Q}\left(\tau^{*} f\right) \mu=\int_{M} f \Omega$. Recall that $\Omega$ is the Liouville volume form on $M$.

With respect to $\mu$ we take the $L^{2}$-completion $\mathrm{L}^{2}(Q, \mu)$ of the space of functions on $Q$. The generalized Hardy space $\mathcal{H}$ is the closure of the functions in $\mathrm{L}^{2}(Q, \mu)$ which can be extended to holomorphic functions on the whole disc bundle $D:=\{\lambda \in U \mid k(\lambda, \lambda) \leq 1\}$. The generalized Szegö projector is the projection

$$
\Pi: \mathrm{L}^{2}(Q, \mu) \rightarrow \mathcal{H}
$$

By the natural circle action the bundle $Q$ is a $S^{1}$-bundle and the tensor powers of $U$ can be viewed as associated line bundles. The space $\mathcal{H}$ is preserved by the $S^{1}$-action. It can be decomposed into eigenspaces $\mathcal{H}=\prod_{m=0}^{\infty} \mathcal{H}^{(m)}$ where $c \in S^{1}$ acts on $\mathcal{H}^{(m)}$ as multiplication by $c^{m}$. The Szegö projector is $S^{1}$ invariant and can be decomposed into its components, the Bergman projectors $\hat{\Pi}^{(m)}: \mathrm{L}^{2}(Q, \mu) \rightarrow \mathcal{H}^{(m)}$.

Sections of $L^{m}=U^{-m}$ can be identified with functions $\psi$ on $Q$ which satisfy the equivariance condition $\psi(c \lambda)=c^{m} \psi(\lambda)$, i.e. which are homogeneous of degree $m$. This identification is given via the map

$$
\gamma_{m}: \mathrm{L}^{2}\left(M, L^{m}\right) \rightarrow \mathrm{L}^{2}(Q, \mu), \quad s \mapsto \psi_{s} \quad \text { where } \quad \psi_{s}(\alpha)=\alpha^{\otimes m}(s(\tau(\alpha))),
$$

which turns out to be an isometry. Recall that $\mathrm{L}^{2}\left(M, L^{m}\right)$ has a scalar product given in an corresponding way to (2.9). Restricted to the holomorphic sections we obtain the isometry

$$
\gamma_{m}: \Gamma_{h o l}\left(M, L^{m}\right) \cong \mathcal{H}^{(m)} .
$$


There is the notion of Toeplitz structure $(\Pi, \Sigma)$ as developed by Boutet de Monvel and Guillemin in [5],[12]. Here $\Pi$ is the Szegö projector (4.1) and $\Sigma$ is the submanifold

$$
\Sigma=\{t \alpha(\lambda) \mid \lambda \in Q, t>0\} \subset T^{*} Q \backslash 0
$$

of the tangent bundle of $Q$ defined with the help of the 1-form $\alpha$. They showed that $\Sigma$ is a symplectic submanifold. A (generalized) Toeplitz operator of order $k$ is an operator $A: \mathcal{H} \rightarrow \mathcal{H}$ of the form $A=\Pi \cdot R \cdot \Pi$ where $R$ is a pseudodifferential operator ( $\Psi D O$ ) of order $k$ on $Q$. The Toeplitz operators build a ring. The symbol of $A$ is the restriction of the principal symbol of $R$ (which lives on $T^{*} Q$ ) to $\Sigma$. Note that $R$ is not fixed by $A$, but Guillemin and Boutet de Monvel showed that the symbols are well-defined and that they obey the same rules as the symbols of $\Psi$ DOs. In particular we have the following relations

$$
\sigma\left(A_{1} A_{2}\right)=\sigma\left(A_{1}\right) \sigma\left(A_{2}\right), \quad \sigma\left(\left[A_{1}, A_{2}\right]\right)=\mathrm{i}\left\{\sigma\left(A_{1}\right), \sigma\left(A_{2}\right)\right\}_{\Sigma} .
$$

Here $\{., .\}_{\Sigma}$ is the restriction of the canonical Poisson structure of $T^{*} Q$ to $\Sigma$ coming from the canonical symplectic form $\omega_{0}$ on $T^{*} Q$.

We have to deal with two Toeplitz operators:

(1) The generator of the circle action gives the operator $D_{\varphi}=\frac{1}{\mathrm{i}} \frac{\partial}{\partial \varphi}$. It is an operator of order 1 with symbol $t$. It operates on $\mathcal{H}^{(m)}$ as multiplication by $m$.

(2) For $f \in C^{\infty}(M)$ let $M_{f}$ be the operator on $\mathrm{L}^{2}(Q, \mu)$ corresponding to multiplication with $\tau^{*} f$. We set ${ }^{1} T_{f}=\Pi \cdot M_{f} \cdot \Pi: \mathcal{H} \rightarrow \mathcal{H}$. Because $M_{f}$ is constant along the fibres of $\tau, T_{f}$ commutes with the circle action. Hence $T_{f}=\prod_{m=0}^{\infty} T_{f}^{(m)}$, where $T_{f}^{(m)}$ denotes the restriction of $T_{f}$ to $\mathcal{H}^{(m)}$. After the identification of $\mathcal{H}^{(m)}$ with $\Gamma_{\text {hol }}\left(M, L^{m}\right)$ we see that these $T_{f}^{(m)}$ are exactly the Toeplitz operators $T_{f}^{(m)}$ introduced in Section 2. In this sense $T_{f}$ is called the global Toeplitz operator and the $T_{f}^{(m)}$ the local Toeplitz operators. $T_{f}$ is an operator of order 0 . Let us denote by $\tau_{\Sigma}: \Sigma \subseteq T^{*} Q \rightarrow Q \rightarrow M$ the composition then we obtain for the symbol $\sigma\left(T_{f}\right)=\tau_{\Sigma}^{*}(f)$.

Now we are able to proof (3.3). The commutator $\left[T_{f}, T_{g}\right]$ is a Toeplitz operator of order -1 . Using $\omega_{0 \mid t \alpha(\lambda)}=-t \tau_{\Sigma}^{*} \omega$ if $t$ is a fixed positive number, we obtain with (4.5) that the principal symbol of the commutator equals

$$
\sigma\left(\left[T_{f}, T_{g}\right]\right)(t \alpha(\lambda))=\mathrm{i}\left\{\tau_{\Sigma}^{*} f, \tau_{\Sigma}^{*} g\right\}_{\Sigma}(t \alpha(\lambda))=-\mathrm{i} t^{-1}\{f, g\}_{M}(\tau(\lambda)) .
$$

Now consider the Toeplitz operator

$$
A:=D_{\varphi}^{2}\left[T_{f}, T_{g}\right]+\mathrm{i} D_{\varphi} T_{\{f, g\}} .
$$

Formally this is an operator of order 1. Using $\sigma\left(T_{\{f, g\}}\right)=\tau_{\Sigma}^{*}\{f, g\}$ and $\sigma\left(D_{\varphi}\right)=t$ we see that its principal symbol vanishes. Hence it is an operator of order 0 . Now $M$ and hence $Q$ are compact manifolds. This implies that $A$ is a bounded operator ( $\Psi D O$ of order 0 on compact manifolds are bounded). It is obviously $S^{1}$-invariant and we can write $A=\prod_{m=0}^{\infty} A^{(m)}$ where $A^{(m)}$ is the restriction of $A$ on the space $\mathcal{H}^{(m)}$. For the norms we get $\left\|A^{(m)}\right\| \leq\|A\|$. But

$$
A^{(m)}=A_{\mid \mathcal{H}^{(m)}}=m^{2}\left[T_{f}^{(m)}, T_{g}^{(m)}\right]+\mathrm{i} m T_{\{f, g\}}^{(m)} .
$$

\footnotetext{
${ }^{1}$ There should be no confusion with the operator $T_{f}=T_{f}^{(1)}$ introduced above.
} 
Taking the norm bound and dividing it by $m$ we get the the statement (3.3).

Quite similar (3.4) and Theorem 3.2 can be proved. The original proof of (3.2) presented in [4] uses different techniques. Below I will introduce the Berezin transform. With the help of its asymptotic expansion shown in [14] I will give further down a different proof of (3.2).

\section{BEREZIN SYMBOLS AND THE BEREZIN TRANSFORM}

5.1. Coherent States. Let the situation as in the previous section. In particular $L$ is assumed to be already very ample, $U=L^{*}$ is the dual of the quantum line bundle, $Q \subset U$ the unit circle bundle, and $\tau: Q \rightarrow M$ the projection. Recall that the sections of $L^{m}$ can be identified with the homogeneous functions on $Q$ of degree $m$, see (4.2). For every element $\alpha \in U \backslash 0$ there exists a unique section $e_{\alpha}^{(m)} \in \Gamma_{\text {hol }}\left(M, L^{m}\right)$ such that

$$
\left\langle s, e_{\alpha}^{(m)}\right\rangle=\psi_{s}(\alpha)=\alpha^{\otimes m}(s(\tau(\alpha)))
$$

for all $s \in \Gamma_{h o l}\left(M, L^{m}\right)$. This section is called the coherent vector associated to the point $\alpha$. Recall that $\langle.,$.$\rangle denotes the scalar product on the space of global sections \Gamma_{\infty}\left(M, L^{m}\right)$. We assume it to be linear in the first argument and anti-linear in the second argument. The definition is dual to the definition of coherent vectors of Berezin in its coordinate independent version and extension due to Rawnsley [16],[7], see also [3],[18]. There the coherent vectors are parameterized by the elements of $L \backslash 0$. Our definition has the advantage that we can consider all tensor powers of $L$ together.

The coherent vectors are antiholomorphic in $\alpha$ and fulfill

$$
e_{c \alpha}^{(m)}=\bar{c} \cdot e_{\alpha}^{(m)}, \quad c \in \mathbb{C}^{*}:=\mathbb{C} \backslash\{0\} .
$$

Note that $e_{\alpha}^{(m)} \equiv 0$ would imply that all sections will vanish at the point $x=\tau(\alpha)$. But this is a contradiction to the very-ampleness of $L$. Hence, $e_{\alpha}^{(m)} \not \equiv 0$ and due to (5.2) the element $\left[e_{\alpha}^{(m)}\right]:=\left\{s \in \Gamma_{h o l}\left(M, L^{m}\right) \mid \exists c \in \mathbb{C}^{*}: s=c \cdot e_{\alpha}^{(m)}\right\}$ is a well-defined element of the projective space $\mathbb{P}\left(\Gamma_{h o l}\left(M, L^{m}\right)\right)$ only depending on $x=\tau(\alpha) \in M$. It is called the coherent state associated to $x \in M$.

The coherent state embedding is the antiholomorphic embedding

$$
M \quad \rightarrow \quad \mathbb{P}\left(\Gamma_{h o l}\left(M, L^{m}\right)\right) \cong \mathbb{P}^{N}(\mathbb{C}), \quad x \mapsto\left[e_{\tau^{-1}(x)}\right] .
$$

In abuse of notation in this context we will understand under $\tau^{-1}(x)$ always a non-zero element of the fiber over $x$. The coherent state embedding is up to conjugation the embedding with respect to an orthonormal basis of the sections. See [1] for further considerations of the geometry involved.

5.2. Berezin symbols. The covariant Berezin symbol $\sigma(A)$ of an operator $A \in \operatorname{End}\left(\Gamma_{h o l}\left(M, L^{(m)}\right)\right)$ is defined as

$$
\sigma(A): M \rightarrow \mathbb{C}, \quad x \mapsto \sigma(A)(x):=\frac{\left\langle A e_{\alpha}^{(m)}, e_{\alpha}^{(m)}\right\rangle}{\left\langle e_{\alpha}^{(m)}, e_{\alpha}^{(m)}\right\rangle}, \quad \alpha \in \tau^{-1}(x), \alpha \neq 0 .
$$

It is a well-defined function on $M$.

It is shown in [21] that the Toeplitz map $f \mapsto T_{f}^{(m)}$ and the symbol map $A \mapsto \sigma(A)$ are adjoint if one takes for the operators the Hilbert-Schmidt norm and for the functions the Liouville measure modified by Rawnsley's epsilon function. Here I will not go into the details 
of the correspondence. Let me only point out that every operator of $\Gamma_{h o l}\left(M, L^{m}\right)$ can be represented as $T_{f}^{(m)}$ with a suitable function $f$ which is in general not unique. This $f$ is also called a contravariant symbol $\check{\sigma}(A)$ of the operator $A$.

Starting from $f \in C^{\infty}(M)$ we can assign to it its Toeplitz operator $T_{f}^{(m)} \in \operatorname{End}\left(\Gamma_{h o l}\left(M, L^{(m)}\right)\right)$ and then assign to $T_{f}^{(m)}$ the covariant symbol $\sigma\left(T_{f}^{(m)}\right)$. It is again an element of $C^{\infty}(M)$. Altogether we obtain a map $f \mapsto I^{(m)}(f):=\sigma\left(T_{f}^{(m)}\right)$.

Definition 5.1. The map

$$
C^{\infty}(M) \rightarrow C^{\infty}(M), \quad f \mapsto I^{(m)}(f):=\sigma\left(T_{f}^{(m)}\right)
$$

is called Berezin transform.

From the point of view of Berezin's approach [2] the operator $T_{f}^{(m)}$ has as a contravariant symbol $f$. Hence $I^{(m)}$ gives a correspondence between contravariant symbols and covariant symbols of operators. The Berezin transform was introduced and studied by Berezin [2] for certain classical symmetric domains in $\mathbb{C}^{n}$. These results where extended by Unterberger and Upmeier [24], see also Engliš [8],[9],[10] and Engliš and Peetre [11]. Obviously, the Berezin transform makes also sense in the compact Kähler case which we consider here.

5.3. Asymptotic expansion of the Berezin transform. The results presented in this subsection are joint work with Alexander Karabegov [14]. Recall from Section 4 the Szegö projectors $\Pi: \mathrm{L}^{2}(Q, \mu) \rightarrow \mathcal{H}$ and its components $\hat{\Pi}^{(m)}: \mathrm{L}^{2}(Q, \mu) \rightarrow \mathcal{H}^{(m)}$, the Bergman projectors. The Bergman projectors have a smooth integral kernels, the Bergman kernels $\mathcal{B}_{m}(\alpha, \beta)$ defined on $Q \times Q$, i.e.

$$
\hat{\Pi}^{(m)}(\psi)(\alpha)=\int_{Q} \mathcal{B}_{m}(\alpha, \beta) \psi(\beta) \mu(\beta) .
$$

The Bergman kernels can be expressed with the help of the coherent vectors.

\section{Proposition 5.2.}

$$
\mathcal{B}_{m}(\alpha, \beta)=\psi_{e_{\beta}^{(m)}}(\alpha)=\overline{\psi_{e_{\alpha}^{(m)}}(\beta)}=\left\langle e_{\beta}^{(m)}, e_{\alpha}^{(m)}\right\rangle .
$$

Proof. First note that for $s \in \mathrm{L}^{2}\left(M, L^{m}\right)$ we have $\hat{\Pi}^{(m)} \gamma_{m}(s)=\gamma_{m} \Pi^{(m)} s$, i.e. $\hat{\Pi}^{(m)} \psi_{s}=$ $\psi_{\Pi^{(m)} s}$. Due to the fact that $e_{\alpha}^{(m)}$ is a holomorphic section

$$
\left\langle s, e_{\alpha}^{(m)}\right\rangle=\left\langle s, \Pi^{(m)} e_{\alpha}^{(m)}\right\rangle=\left\langle\Pi^{(m)} s, e_{\alpha}^{(m)}\right\rangle=\psi_{\Pi^{(m)} s}(\alpha)=\hat{\Pi}^{(m)} \psi_{s}(\alpha)=\int_{Q} \mathcal{B}_{m}(\alpha, \beta) \psi_{s}(\beta) \mu(\beta)
$$

By the isometry

$$
\left\langle s, e_{\alpha}^{(m)}\right\rangle=\left\langle\psi_{s}, \psi_{e_{\alpha}^{(m)}}\right\rangle=\int_{Q} \psi_{s}(\beta) \overline{\psi_{e_{\alpha}^{(m)}}(\beta)} \mu(\beta) .
$$

If we compare this two expressions and take the definition of the coherent vectors we obtain

$$
\mathcal{B}_{m}(\alpha, \beta)=\overline{\psi_{e_{\alpha}^{(m)}}(\beta)}=\overline{\left\langle e_{\alpha}^{(m)}, e_{\beta}^{(m)}\right\rangle}=\left\langle e_{\beta}^{(m)}, e_{\alpha}^{(m)}\right\rangle=\psi_{e_{\beta}^{(m)}}(\alpha) .
$$


Let $x, y \in M$ and choose $\alpha, \beta \in Q$ with $\tau(\alpha)=x$ and $\tau(\beta)=y$ then the functions

$$
\begin{gathered}
u_{m}(x):=\mathcal{B}_{m}(\alpha, \alpha)=\left\langle e_{\alpha}^{(m)}, e_{\alpha}^{(m)}\right\rangle \\
v_{m}(x, y):=\mathcal{B}_{m}(\alpha, \beta) \cdot \mathcal{B}_{m}(\beta, \alpha)=\left\langle e_{\beta}^{(m)}, e_{\alpha}^{(m)}\right\rangle \cdot\left\langle e_{\alpha}^{(m)}, e_{\beta}^{(m)}\right\rangle
\end{gathered}
$$

are well-defined on $M$. The following proposition gives an integral representation of the Berezin transform.

\section{Proposition 5.3.}

$$
\begin{aligned}
\left(I^{(m)}(f)\right)(x) & =\frac{1}{\mathcal{B}_{m}(\alpha, \alpha)} \int_{Q} \mathcal{B}_{m}(\alpha, \beta) \mathcal{B}_{m}(\beta, \alpha) \tau^{*} f(\beta) \mu(\beta) \\
& =\frac{1}{u_{m}(x)} \int_{M} v_{m}(x, y) f(y) \Omega(y)
\end{aligned}
$$

Proof. Take any $\alpha \in \tau^{-1}(x)$ with $\alpha \in Q$. Denote by $M_{f}$ the operator of pointwise multiplication of the sections with the function $f$.

$$
\begin{aligned}
\left(I^{(m)} f\right)(x) & =\sigma\left(T_{f}^{(m)}\right)(x)=\frac{\left\langle T_{f}^{(m)} e_{\alpha}^{(m)}, e_{\alpha}^{(m)}\right\rangle}{\left\langle e_{\alpha}^{(m)}, e_{\alpha}^{(m)}\right\rangle} \\
& =\frac{\left\langle\Pi^{(m)} M_{f} \Pi^{(m)} e_{\alpha}^{(m)}, e_{\alpha}^{(m)}\right\rangle}{\mathcal{B}_{m}(\alpha, \alpha)}=\frac{\left\langle M_{f} e_{\alpha}^{(m)}, e_{\alpha}^{(m)}\right\rangle}{\mathcal{B}_{m}(\alpha, \alpha)}
\end{aligned}
$$

Using the isometry (4.2) and Proposition 5.2 we can rewrite the last expression and obtain

$$
\begin{aligned}
\left(I^{(m)} f\right)(x) & =\frac{\left\langle\left(\tau^{*} f\right) \psi_{e_{\alpha}^{(m)}}, \psi_{e_{\alpha}^{(m)}}\right\rangle}{\mathcal{B}_{m}(\alpha, \alpha)}=\frac{1}{\mathcal{B}_{m}(\alpha, \alpha)} \int_{Q}\left(\tau^{*} f\right) \psi_{e_{\alpha}^{(m)}}(\beta) \overline{\psi_{e_{\alpha}^{(m)}}^{(m)}} \mu(\beta) \\
& =\frac{1}{\mathcal{B}_{m}(\alpha, \alpha)} \int_{Q} \mathcal{B}_{m}(\alpha, \beta) \mathcal{B}_{m}(\beta, \alpha)\left(\tau^{*} f\right)(\beta) \mu(\beta)=\frac{1}{u_{m}(x)} \int_{M} v_{m}(x, y) f(y) \Omega(y)
\end{aligned}
$$

In [14] based on works of Boutet de Monvel and Sjöstrand [6] on the Szegö kernel and in generalization of a result of Zelditch [26] on the Bergman kernel the integral representation is used to prove the existence and the form of the asymptotic expansion of the Berezin transform. It can be identified with a formal Berezin transform introduced by A. Karabegov. Karabegov developed a theory for such formal deformation quantizations of (pseudo-) Kähler manifolds which fulfill the "separation of variables" properties. In particular, he assigns to every such deformation quantization a formal Berezin transform $I$. It is a formal power series in the variable $\nu$ and can be written as $I=\sum_{j=0}^{\infty} I_{j} \nu^{j}$ with operators $I_{j}: C^{\infty}(M) \rightarrow C^{\infty}(M)$. He proved that it starts with $I=i d+\nu \Delta+\nu^{2} \cdots$. In [14] it is shown that if we replace $\frac{1}{m}$ by the formal variable $\nu$ in the asymptotic expansion of the Berezin transform $I^{(m)} f(x)$ we obtain the formal Berezin transform $I(f)(x)$ with respect to a specified star product (Theorem 5.9 in [14]). In particular, we obtain for the asymptotic expansion of the Berezin transform at a 
fixed point $x \in M$

$$
I^{(m)} f(x) \sim f(x)+\frac{1}{m} \Delta f(x)+\cdots, \quad \text { for } m \rightarrow \infty .
$$

Some details and intermediate results, e.g. a stationary phase integral (A.7) which is asymptotically equivalent to $\left(I^{(m)} f\right)(x)$, are given in an appendix to this write-up.

\section{Applications of the asymptotics of the Berezin transform}

6.1. Norm preservation. Here I like to prove that Relation (3.2) in Theorem 3.1 can be easily deduced from (5.13). First note that

$$
\left\|I^{(m)}(f)\right\|_{\infty}=\left\|\sigma\left(T_{f}^{(m)}\right)\right\|_{\infty} \leq\left\|T_{f}^{(m)}\right\| \leq\|f\|_{\infty} .
$$

This has been shown in [21]. For the convenience of the reader I will repeat the proof here. Using Cauchy-Schwarz inequality we calculate $(x=\tau(\alpha))$

$$
\left|\sigma\left(T_{f}^{(m)}\right)(x)\right|^{2}=\frac{\left|\left\langle T_{f}^{(m)} e_{\alpha}^{(m)}, e_{\alpha}^{(m)}\right\rangle\right|^{2}}{\left\langle e_{\alpha}^{(m)}, e_{\alpha}^{(m)}\right\rangle^{2}} \leq \frac{\left\langle T_{f}^{(m)} e_{\alpha}^{(m)}, T_{f}^{(m)} e_{\alpha}^{(m)}\right\rangle}{\left\langle e_{\alpha}^{(m)}, e_{\alpha}^{(m)}\right\rangle} \leq\left\|T_{f}^{(m)}\right\|^{2} .
$$

Here the last inequality follows from the definition of the operator norm. This shows the first inequality in (6.1). For the second inequality introduce the multiplication operator $M_{f}^{(m)}$ on $\Gamma_{\infty}\left(M, L^{m}\right)$. Then $\left\|T_{f}^{(m)}\right\|=\left\|\Pi^{(m)} M_{f}^{(m)} \Pi^{(m)}\right\| \leq\left\|M_{f}^{(m)}\right\|$ and for $\varphi \in \Gamma_{\infty}\left(M, L^{m}\right), \varphi \neq 0$

$$
\frac{\left\|M_{f}^{(m)} \varphi\right\|^{2}}{\|\varphi\|^{2}}=\frac{\int_{M} h^{(m)}(f \varphi, f \varphi) \Omega}{\int_{M} h^{(m)}(\varphi, \varphi) \Omega}=\frac{\int_{M} f(z) \overline{f(z)} h^{(m)}(\varphi, \varphi) \Omega}{\int_{M} h^{(m)}(\varphi, \varphi) \Omega} \leq\|f\|_{\infty}^{2} .
$$

Hence,

$$
\left\|T_{f}^{(m)}\right\| \leq\left\|M_{f}^{(m)}\right\|=\sup _{\varphi \neq 0} \frac{\left\|M_{f}^{(m)} \varphi\right\|}{\|\varphi\|} \leq\|f\|_{\infty}
$$

Now choose as $x_{e} \in M$ a point with $\left|f\left(x_{e}\right)\right|=\|f\|_{\infty}$. From the fact that the formal Berezin transform has as leading term the identity it follows that $\left|\left(I^{(m)} f\right)\left(x_{e}\right)-f\left(x_{e}\right)\right| \leq C / m$ with a suitable constant $C$. This implies ||$f\left(x_{e}\right)|-|\left(I^{(m)} f\right)\left(x_{e}\right)|| \leq C / m$ and hence

$$
\|f\|_{\infty}-\frac{C}{m}=\left|f\left(x_{e}\right)\right|-\frac{C}{m} \leq\left|\left(I^{(m)} f\right)\left(x_{e}\right)\right| \leq\left\|I^{(m)} f\right\|_{\infty} .
$$

Putting (6.1) and (6.5) together we obtain

$$
\|f\|_{\infty}-\frac{C}{m} \leq\left\|T_{f}^{(m)}\right\| \leq|f|_{\infty} .
$$

Note that we obtain in this way another proof of [4], Theorem 4.1. 
6.2. Pullback of the Fubini-Study form. Starting from the Kähler manifold $(M, \omega)$ and after choosing an orthonormal basis of the space $\Gamma_{h o l}\left(M, L^{m}\right)$ we obtain an embedding $\phi^{(m)}$ : $M \rightarrow \mathbb{P}^{N(m)}$ of $M$ into projective space of dimension $N(m)$. On $\mathbb{P}^{N(m)}$ we have the standard Kähler form, the Fubini-Study form $\omega_{F S}$. The pull-back $\left(\phi^{(m)}\right)^{*} \omega_{F S}$ will not depend on the orthogonal basis chosen for the embedding but in general it will not coincide with a scalar multiple of the Kähler form $\omega$ we started with (see [1] for a thorough discussion of the situation). It was shown by Zelditch [26] by generalizing a result of Tian that $\left(\Phi^{(m)}\right)^{*} \omega_{F S}$ admits a complete asymptotic expansion in powers of $\frac{1}{m}$ as $m \rightarrow \infty$.

The Karabegov classification of star products with the "separation of variables" property for $(M, \omega)$ can be given by assigning to it a unique formal form $\widehat{\omega}=\frac{1}{\nu} \omega+\omega_{0}+\nu \omega_{1}+\cdots$ where the $\omega_{i}$ for $i \in \mathbb{N}_{0}$ are closed $(1,1)$-forms on $M$. From the proof of the identification of the Berezin transform with the formal Berezin transform assigned to a certain star product $\star$, it follows that the classifying form of of exactly this $\star$ coincides with the form obtained via the asymptotic expansion of $\left(\phi^{(m)}\right)^{*} \omega_{F S}$ if one replaces $\frac{1}{m}$ by $\nu$ (see also the appendix). Let me add that in Karabegov's theory the "opposite of the dual star product" to $\star$ is the Berezin-Toeplitz star product $\star_{B T}$ of Theorem 3.2.

\section{Some Comments on the DYNAMics}

First note that we have the following proposition.

Proposition 7.1. The $T_{f}^{(m)} \in \operatorname{End}\left(\Gamma_{\text {hol }}\left(M, L^{(m)}\right)\right)$ fulfill the relation

$$
T_{f}^{(m)^{*}}=T_{\bar{f}}^{(m)} \text {. }
$$

Proof. Let $s, t \in \Gamma_{\text {hol }}\left(M, L^{m}\right)$. For the scalar product we calculate

$$
\left\langle s, T_{f}^{(m)} t\right\rangle=\left\langle s, \Pi^{(m)} f t\right\rangle=\langle s, f t\rangle=\langle\bar{f} s, t\rangle=\left\langle T_{\bar{f}}^{(m)} s, t\right\rangle .
$$

Due to the fact that the identification of $\Gamma_{h o l}\left(M, L^{m}\right)$ with $\mathcal{H}^{(m)}$ is an isometry, the Equation (7.1) is also true for the components of the global Toeplitz operator. In particular, we have $T_{f}{ }^{*}=T_{\bar{f}}$. This implies that for real-valued functions $f$ on $M$ (e.g. for a Hamiltonian) the quantum operators $T_{f}$ and $T_{f}^{(m)}$ are self-adjoint operators.

From the theory of generalized Toeplitz operators follows some consequences for the spectral asymptotics of these operators. Set $d(m):=\operatorname{dim} \mathcal{H}^{(m)}$ and let $\lambda_{1}^{(m)}, \lambda_{2}^{(m)}, \ldots, \lambda_{d(m)}^{(m)}$ be the eigenvalues of the restriction of $T_{f}$ on $\mathcal{H}^{(m)}$. In particular, these are also the eigenvalues of $T_{f}^{(m)}$ on $\Gamma_{h o l}\left(M, L^{m}\right)$. Following [5] $\left(n=\operatorname{dim}_{\mathbb{C}} M\right)$ let

$$
\rho_{m}:=\frac{1}{m^{n}} \sum_{i=1}^{d(m)} \delta\left(\lambda-\lambda_{i}^{(m)}\right)
$$

be the discrete spectral measure. By Theorem 13.13 of [5] it converges weakly to the limit measure

$$
\rho(g)=\gamma_{M} \int_{M} g(f(z)) \Omega(z)
$$


with an universal constant $\gamma_{M}$ only depending on the manifold $M$. For $g \equiv 1$ we obtain

$$
\frac{1}{m^{n}} \sum_{i=1}^{d(m)} \lambda_{i}^{(m)}=\frac{1}{m^{n}} \operatorname{Tr}^{(m)} T_{f}^{(m)}=\gamma_{M} \int_{M} f \Omega+O\left(\frac{1}{m}\right) .
$$

Here $\operatorname{Tr}^{(m)}$ denotes the trace on $\operatorname{End}\left(\Gamma_{h o l}\left(M, L^{m}\right)\right)$. The constant evaluates to $\gamma_{M}=\operatorname{vol}\left(\mathbb{P}^{n}(\mathbb{C})\right)^{-1}$. By linearity this extends to complex-valued functions. Hence,

Proposition 7.2. [4] Let $f \in C^{\infty}(M)$ and let $n=\operatorname{dim}_{\mathbb{C}} M$. Then

$$
\operatorname{Tr}^{(m)}\left(T_{f}^{(m)}\right)=m^{n}\left(\frac{1}{\operatorname{vol}\left(\mathbb{P}^{n}(\mathbb{C})\right)} \int_{M} f \Omega+O\left(m^{-1}\right)\right)
$$

Indeed a closer analysis (i.e. the application of Equation 13.13 in [5]) shows that $\operatorname{Tr}^{(m)}\left(T_{f}^{(m)}\right)$ admits a complete asymptotic expansion which allows to construct a formal trace for the Berezin-Toeplitz star product [22].

Of course, now the question arrises how to quantize symplectic maps $\Psi$ of the phase-space (Kähler) manifold $(M, \omega)$. A first condition is that the map lifts to a contact transformation $\hat{\Psi}$ of the $S^{1}$-bundle $(Q, \alpha)$. Note that $d \alpha=\tau^{*} \omega$ and $(Q, \alpha)$ is a contact structure. Let $L_{\hat{\Psi}}$ be the translation operator by $\Psi$ then the Toeplitz operator $\Pi L_{\hat{\Psi}} \Pi$ is not necessarily a unitary operator. But it was shown by Zelditch [25] that there exists always a function $f \in C^{\infty}(M)$, such that

$$
U_{\hat{\Psi}}=\Pi M_{f} L_{\hat{\Psi}} \Pi
$$

is unitary and commutes with the $S^{1}$ action. In particular, it decomposes again as

$$
U_{\hat{\Psi}}=\prod_{i=0}^{\infty} U_{\hat{\Psi}}^{(m)}
$$

where each $U_{\hat{\Psi}}^{(m)}$ is an unitary operator on $\mathcal{H}^{(m)} \cong \Gamma_{h o l}\left(M, L^{m}\right)$. Such kind of maps where studied by Zelditch [25]. Further work is in progress.

\section{ApPENDix A. MORE DETAILS ON THE ASYMPtotic EXPANSION}

In this appendix I will express the integral formula (5.10) of the Berezin transform up to asymptotic equivalence as a stationary phase integral (A.7). Clearly, here the steps can only be sketched. For a complete derivation, see [14]. We start with the formula (5.10) and fix a point $x \in M$. Let $W$ be a small contractible neighbourhood of $x$. Split $M$ into the two subsets $W$ and $M \backslash W$ and correspondingly the integral. From results of Boutet de Monvel and Sjöstrand [6] it follows that for $y \in M \backslash W$ the Bergman kernel $\mathcal{B}_{m}(x, y)$ is rapidly decreasing for $m \rightarrow \infty$. For the Bergman kernel on the diagonal Zelditch [26] proved that $u_{m}(x)=\mathcal{B}_{m}(x, x)$ expands in an asymptotic series

$$
u_{m}(x) \sim m^{n} \sum_{r \geq 0}\left(\frac{1}{m}\right)^{r} b_{r}(x), \quad \text { with } b_{0}=1, \quad \text { for } m \rightarrow \infty .
$$

Hence, the integral over $M \backslash W$ will be rapidly decreasing as $m \rightarrow \infty$ (here the compactness of $M$ is used). It follows that up to asymptotic equivalence it is enough to consider the integral 
over $W$. In the following I will use almost analytic extensions. Without too much misconception the reader might imagine almost analytic extensions similar to analytic extensions of a real-analytic object $a(x)$ defined on $W$ to an object $\tilde{a}(x, y)$ on $W \times W$ which is holomorphic in the first variable, antiholomorphic in the second, and fulfills $\tilde{a}(x, x)=a(x)$ on $W$. Such an almost analytic expansion exists for every $C^{\infty}$-function.

Let $\Phi$ be the (local) Kähler potential of $\omega$, i.e. $\omega=-\mathrm{i} \partial \bar{\partial} \Phi$, over $W$ (shrinking $W$ if necessary) and $\tilde{\Phi}$ an almost analytic extension fulfilling $\tilde{\Phi}(y, x)=\overline{\tilde{\Phi}(x, y)}$. Set

$$
\chi(x, y):=\tilde{\Phi}(x, y)-\frac{1}{2} \Phi(x)--\frac{1}{2} \Phi(y) \text { and } D(x, y):=\chi(x, y)+\chi(y, x) .
$$

Let $e$ be a local holomorphic frame of $U$ over $W$. For any $x \in W$ take

$$
\alpha(x):=\frac{e(x)}{\sqrt{k(e(x), e(x))}}
$$

as corresponding point in $Q_{\mid W}$. Now by Theorem 5.6 of [14] we obtain that there exists an asymptotic expansion of the Bergman kernel over $W \times W$ for $m \rightarrow \infty$

$$
\mathcal{B}_{m}(\alpha(x), \alpha(y)) \sim m^{n} \mathrm{e}^{m \chi(x, y)} \sum_{r \geq 0}\left(\frac{1}{m}\right)^{r} \tilde{b}_{r}(x, y),
$$

where the $\tilde{b}_{r}(x, y)$ are almost analytic extensions of the $b_{r}(x)$ which are given by (A.1). Let $b(x, y, m) \in S^{0}(W \times W \times \mathbb{R})$ be a symbol such that it has the asymptotic expansion

$$
b(x, y, m) \sim \sum_{r \geq 0}\left(\frac{1}{m}\right)^{r} \tilde{b}_{r}(x, y) .
$$

We conclude from (A.4) that we have the asymptotic expansion of the two-point function

$$
v_{m}(x, y) \sim m^{2 n} e^{m D(x, y)} b(x, y, m) b(y, x, m)
$$

Now $\phi^{x}(y):=D(x, y)$ are phase functions such that $y=x$ are nondegenerate isolated critical points. Again by shrinking $W$ if necessary, we can achieve that $y=x$ are the only critical points and that $\tilde{b}_{0}(x, y)$ does not vanish on the closure of $W \times W$. We obtain the following asymptotic equivalence

$$
\left(I^{m} f\right)(x) \sim m^{n} \int_{W} e^{m \phi^{x}(y)} f(y) \frac{b(x, y, m) b(y, x, m)}{b(x, x, m)} \Omega(y) .
$$

This is a stationary phase integral. From this follows the asymptotic expansion of the Berezin transform and after some additional work [14] its identification with the formal Berezin transform associated to a certain formal star product $\star$.

Let me indicate some steps necessary to identify the star product $\star$. If we replace in $\sum_{r \geq 0}\left(\frac{1}{m}\right)^{r} b_{r}$ from (A.1) the expression $\frac{1}{m}$ by the formal variable $\nu$ we can find another formal function $s$ such that $\sum_{r \geq 0} \nu^{r} b_{r}(x)=\mathrm{e}^{s(x)}$. Note that $b_{0}(x) \equiv 1$. Now we set $\widehat{\Phi}:=\frac{1}{\nu} \Phi+s$ (again a formal power series). It turns out that the Karabegov form $\tilde{\omega}$ classifying the star product $\star$ is given by $\widehat{\omega}=-\mathrm{i} \partial \bar{\partial} \widehat{\Phi}$. The form $\tilde{\omega}$ is a formal Laurent series in the variable $\nu$

$$
\widehat{\omega}=\frac{1}{\nu} \omega+\sum_{j=0}^{\infty} \nu^{j} \omega_{j}
$$


where $\omega$ is the Kähler form we started with and the $\omega_{i}$ are global closed $(1,1)$-forms (not necessarily nondegenerate).

As is shown in [14] the Karabegov classifying formal form $\omega_{B T}^{\prime}$ of the opposite of the Berezin-Toeplitz star product $\star_{B T}$ is

$$
\omega_{B T}^{\prime}=-\frac{1}{\nu} \omega+\omega_{c a n}
$$

where $\omega_{\text {can }}$ is the curvature form of the canonical holomorphic line bundle of $M$ with fibre metric given by the metric coming from the Liouville form. The opposite star product $\star_{B T}^{\prime}$ is defined as $f \star_{B T}^{\prime} g:=g \star_{B T} f$. This switches the rôles played by the holomorphic and antiholomorphic functions in the definition of "separation of variables" in accordance with its use by Karabegov [13].

Finally, we conclude

$$
c l\left(\star_{B T}\right)=\frac{1}{\mathrm{i}}\left(\frac{1}{\nu}[\omega]-\frac{\epsilon}{2}\right)
$$

for the characteristic class of the star product $\star_{B T}$, where $\epsilon$ is the canonical class of the manifold $M$.

Let me indicate how the result on the asymptotic of the pull-back of Fubini-Study form presented in Section 6.2 follows from the above presented steps. The pull-back can be given as [26, Prop.9]

$$
\left(\phi^{(m)}\right)^{*} \omega_{F S}=m \omega+\mathrm{i} \partial \bar{\partial} \log u_{m}(x) \text {. }
$$

By the asymptotic expansion (A.1) of $u_{m}(x)$ in terms of the $b_{r}$ due to Zelditch, and the definition of the formal Kähler potential $\widehat{\Phi}$ for the classifying form $\widehat{\omega}$ via the formal function $s$ which is defined above via the $b_{r}$, we conclude that $\widehat{\omega}$ can be obtained from the asymptotic expansion of $\left(\phi^{(m)}\right)^{*} \omega_{F S}$ if one replaces $\frac{1}{m}$ by the formal variable $\nu$.

\section{REFERENCES}

[1] St. Berceanu and M. Schlichenmaier, Coherent state embeddings, polar divisors and Cauchy formulas, JGP 34 (2000), 336-358, math.QA/9902066.

[2] F.A. Berezin, Quantization in complex bounded domains, Soviet Math. Dokl. 14 (1973), 1209-1213.

[3] M. Bordemann, Hoppe J., P. Schaller, and M. Schlichenmaier, $g l(\infty)$ and geometric quantization, Commun. Math. Phys. 138 (1991), 209-244.

[4] M. Bordemann, E. Meinrenken, and M. Schlichenmaier, Toeplitz quantization of Kähler manifolds and $g l(n), n \rightarrow \infty$ limits, Commun. Math. Phys. 165 (1994), 281-296.

[5] L. Boutet de Monvel and V. Guillemin, The spectral theory of Toeplitz operators. Ann. Math. Studies, Nr.99, Princeton University Press, Princeton, 1981.

[6] L. Boutet de Monvel and J. Sjöstrand, Sur la singularite des noyaux de Bergman et de Szegö, Asterisque 34-35 (1976), 123-164.

[7] M. Cahen, S. Gutt, and J. Rawnsley, Quantization of Kähler manifolds I: Geometric interpretation of Berezin's quantization, JGP 7 (1990), 45-62.

[8] M. Englis, Asymptotics of the Berezin transform and quantization on planar domains, Duke Math. J. 79 (1995), 57-76.

[9] M. Englis, Berezin quantization and reproducing kernels on complex domains, Trans. Amer. Math. Soc. 348 (1996), 411-479.

[10] M. Englis, Weighted Bergman kernels and quantization, Preprint, September 2000. 
[11] M. Englis and J. Peetre, On the correspondence principle for the quantized annulus, Math. Scand. 78 (1996), 183-206.

[12]. V. Guillemin, Some classical theorems in spectral theory revisited, Seminars on singularities of solutions of linear partial differential equations, Ann. Math. Studies, Nr.91 (L. Hörmander, ed.), Princeton University Press, 1979, pp. 219-259.

[13] Karabegov, A.: Deformation quantization with separation of variables on a Kähler manifold, Commun. Math. Phys. 180 (1996), 745-755.

[14] A. Karabegov and M. Schlichenmaier, Identification of Berezin-Toeplitz deformation quantization, math.QA/0006063, Mannheimer Manuskripte Nr. 253.

[15] N.P. Landsman, Mathematical topics between classical and quantum mechanics, Springer, Berlin, Heidelberg, New York, 1998.

[16] J. H. Rawnsley, Coherent states and Kähler manifolds, Quart. J. Math. Oxford Ser.(2) 28 (1977), $403-415$.

[17] M. Rieffel, Questions on quantization, Operator algebras and operator theory. Shanghai, China, July 4-9, 1997 (Liming et al., ed.), AMS, 1998, pp. 315-326.

[18] M. Schlichenmaier, Zwei Anwendungen algebraisch-geometrischer Methoden in der theoretischen Physik: Berezin-Toeplitz-Quantisierung und globale Algebren der zweidimensionalen konformen Feldtheorie, Habilitation thesis, 1996.

[19] M. Schlichenmaier, Deformation quantization of compact Kähler manifolds via Berezin-Toeplitz operators, Proceedings of the XXI Int. Coll. on Group Theoretical Methods in Physics (15-20 July 1996, Goslar, Germany) (H.-D. Doebner, P. Nattermann, and W. Scherer, eds.), World Scientific, 1997, pp. 396-400.

[20] M. Schlichenmaier, Berezin-Toeplitz quantization of compact Kähler manifolds, Quantization, Coherent States and Poisson Structures, Proc. XIV'th Workshop on Geometric Methods in Physics (Białowieża, Poland, 9-15 July 1995) (A. Strasburger, S.T. Ali, J.-P. Antoine, J.-P. Gazeau, and A. Odzijewicz, eds.), Polish Scientific Publisher PWN, 1998, q-alg/9601016, pp. 101-115.

[21] M. Schlichenmaier, Berezin-Toeplitz quantization and Berezin symbols for arbitrary compact Kähler manifolds, to appear in the Proceedings of the XVII Bialowieza workshop on Geometric Methods in Physics, math.QA/9902066.

[22] M. Schlichenmaier, Deformation quantization of compact Kähler manifolds by Berezin-Toeplitz quantization, Conference Moshé Flato 1999 (September 1999, Dijon, France) (G. Dito and D. Sternheimer, eds.), Kluwer, 2000, math.QA/9910137, Vol. 2, pp. 289-306.

[23] G.M. Tuynman, Quantization: Towards a comparison between methods, Jour. Math. Phys. 28 (1987), 2829-2840.

[24] A. Unterberger and H. Upmeier, The Berezin transform and invariant differential operators, Commun. Math. Phys. 164 (1994), 563-597.

[25] S. Zelditch, Index and dynamics of quantized contact transformations, Ann. Inst. Fourier (Grenoble) 47 (1997), 305-363.

[26] S. Zelditch, Szegö kernels and a theorem of Tian, Int. Math. Res. Not. 6 (1998), 317-331.

(Martin Schlichenmaier) Department of Mathematics and Computer Science, University of Mannheim, Seminargebäude A5, D-68131 Mannheim, Germany

E-mail address: schlichenmaier@math.uni-mannheim.de 